Pacific Journal of Mathematics

A MEASURE OF THE NONMONOTONICITY OF THE EULER 


\title{
A MEASURE OF THE NONMONOTONICITY OF THE EULER PHI FUNCTION
}

\author{
Harold G. Diamond and Paul ERdös
}

1. Introduction. Let $f$ be a real valued arithmetic function satisfying $\lim _{n \rightarrow \infty} f(n)=+\infty$. Define another arithmetic function $F=F_{f}$ by setting

$$
F_{f}(n)=\sharp\{j<n: f(j) \geqq f(n)\}+\#\{j>n: f(j) \leqq f(n)\} .
$$

The size of the values assumed by the function $F$ provides a measure of the nonmonotonicity of $f$. In particular, $F$ is identically zero if and only if $f$ is strictly increasing.

Here we shall take $f$ to be $\varphi$, Euler's function, and study the associated function $F_{\varphi}$, which we henceforth call $F$.

We shall show that $F(n) / n$ is asymptotically representable as a function of $\varphi(n) / n$. Then we shall prove that $F(n) / n$ has a distribution function. We shall study $\max _{n \leqq x} F(n)$ and $\min _{n>x} F(n)$ and investigate conditions on $\varphi(n) / n$ which lead to large and small values of $F(n) / n$.

We express our thanks to Professor Carl Pomerance for a number of helpful comments and suggestions, and to Dr. Charles R. Wall for his unpublished data on the density function of Euler's function.

2. An asymptotic formula for $F$. For $0 \leqq a, b \leqq \infty$, let

$$
\Phi(a, b)=\#\{n \leqq a: \varphi(n) \leqq b\} \text {. }
$$

We have

$$
\begin{aligned}
& \sharp\{j<n: \varphi(j) \geqq \varphi(n)\}=n-\Phi(n, \varphi(n))+\#\{j<n: \varphi(j)=\varphi(n)\}, \\
& \sharp\{j>n: \varphi(j) \leqq \varphi(n)\}=\Phi(\infty, \varphi(n))-\Phi(n, \varphi(n)) .
\end{aligned}
$$

Thus

$$
F(n)=n+\Phi(\infty, \varphi(n))-2 \Phi(n, \varphi(n))+\#\{j<n: \varphi(j)=\varphi(n)\} .
$$

It is known that

$$
\Phi(\infty, y)=\zeta y+O\left(y e^{-\sqrt{\log y}}\right),
$$

where $\zeta$ denotes the constant $\zeta(2) \zeta(3) / \zeta(6) \approx 1.9436[1]$; and

$$
\Phi(x, y)=x g(y / x)+O\left(y e^{-\sqrt{\log y}}\right),
$$

where $g$ is a continuous, increasing function on $[0,1]$ which is determined by a contour integral [2]. 
Moreover, $g$ is strictly concave, as we now indicate. We have from [2, Eq. (12)] that

$$
\alpha g^{\prime}(\alpha)=g(\alpha)-D_{\varphi}(\alpha), \quad 0<\alpha \leqq 1 .
$$

Here

$$
D_{\varphi}(\alpha)=\lim _{x \rightarrow \infty} \frac{1}{x} \sharp\{n \leqq x: \varphi(n) \leqq \alpha n\} .
$$

It is known that this limit exists and defines a continuous function of $\alpha$ (cf. [6, Ch 4], [7, $\S 5]$ ). Clearly $D_{\varphi}$ is nondecreasing. In fact, it is known to be strictly increasing on $(0,1)[8, \mathrm{pp} .319,323]$.

If we integrate the differential equation for $g$ and use the fact that $g(1)=1$, we obtain

$$
g(\alpha)=\alpha+\alpha \int_{\alpha}^{1} t^{-2} D_{\varphi}(t) d t
$$

and differentiating again, and differencing, we get for $0<u<v \leqq 1$

$$
\begin{aligned}
g^{\prime}(v)-g^{\prime}(u) & =-\frac{1}{v} D_{\varphi}(v)+\frac{1}{u} D_{\varphi}(u)-\int_{u}^{v} t^{-2} D_{\varphi}(t) d t \\
& =-\int_{u}^{v} t^{-1} d D_{\varphi}(t)<\left\{D_{\varphi}(u)-D_{\varphi}(v)\right\} / v<0 .
\end{aligned}
$$

Thus $g$ is strictly concave on $(0,1)$.

Noting that

$$
\begin{aligned}
\sharp\{j<n: \varphi(j)=\varphi(n)\} & \leqq \Phi(\infty, \varphi(n))-\Phi(\infty, \varphi(n)-1) \\
& =O\left\{\varphi(n) e^{-\sqrt{\log \varphi(n)}}\right\},
\end{aligned}
$$

we have

$$
\frac{F(n)}{n}=1+\zeta \frac{\varphi(n)}{n}-2 g\left(\frac{\varphi(n)}{n}\right)+O\left\{\frac{\varphi(n)}{n} e^{-\sqrt{\log \varphi(n)}}\right\} .
$$

If we set

$$
h(u)=1+\zeta u-2 g(u)
$$

and enlarge the error we obtain the asymptotic formula

$$
\frac{F(n)}{n}=h(\phi(n) / n)+O\left(e^{-\sqrt{\log n}}\right) .
$$

Below is an approximate graph of $h$. Note that $h$ is strictly convex. 


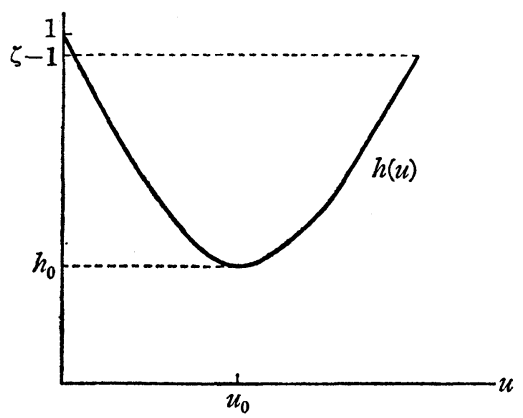

FIGURE 1

\section{A distribution function.}

THEOREM 1. $F(n) / n$ has a continuous distribution function.

Proof. Let $h_{0}$ denote the minimal value of $h$ and $u_{0}$ the point at which the minimum is achieved. Let $h^{*}$ denote the branch of the inverse function of $h$ which maps $\left[h_{0}, 1\right]$ onto $\left[0, u_{0}\right]$, and let $h^{* *}$ denote the branch which maps $\left[h_{0}, \zeta-1\right]$ onto $\left[u_{0}, 1\right]$. Also, let $h^{* *}(\alpha)=1$ for $\zeta-1<\alpha \leqq 1$. Note that $h^{*}$ and $h^{* *}$ are well defined, even at $u_{0}$, on account of the strict convexity of $h$.

Since $D_{\varphi}$ and $h$ are continuous, for $h_{0} \leqq \alpha \leqq 1$ we have

$$
\begin{aligned}
D_{\varphi}\left(h^{* *}(\alpha)\right)-D_{\varphi}\left(h^{*}(\alpha)\right) & =\lim _{x \rightarrow \infty} \frac{1}{x} \sharp\left\{n \leqq x: h^{*}(\alpha) \leqq \varphi(n) / n \leqq h^{* *}(\alpha)\right\} \\
& =\lim _{x \rightarrow \infty} \frac{1}{x} \sharp\{n \leqq x: h(\varphi(n) / n) \leqq \alpha\},
\end{aligned}
$$

a continuous function of $\alpha$ which vanishes at $\alpha=h_{0}$ and equals 1 for $\alpha=1$.

Given $\varepsilon>0$ we have

$$
\begin{aligned}
& \lim _{x \rightarrow \infty} \frac{1}{x} \sharp\left\{n \leqq x: h\left(\frac{\varphi(n)}{n}\right) \leqq \alpha-\varepsilon\right\} \leqq \lim _{x \rightarrow \infty} \frac{1}{x} \sharp\left\{n \leqq x: \frac{F(n)}{n} \leqq \alpha\right\} \\
& \leqq \varlimsup_{x \rightarrow \infty} \frac{1}{x} \#\left\{n \leqq x: \frac{F(n)}{n} \leqq \alpha\right\} \leqq \lim _{x \rightarrow \infty} \frac{1}{x} \#\left\{n \leqq x: h\left(\frac{\varphi(n)}{n}\right) \leqq \alpha+\varepsilon\right\} .
\end{aligned}
$$

It follows that if $h_{0} \leqq \alpha \leqq 1$, then

$$
D_{F}(\alpha)=\lim _{x \rightarrow \infty} \frac{1}{x} \sharp\left\{n \leqq x: \frac{F(n)}{n} \leqq \alpha\right\}=D_{\varphi}\left(h^{* *}(\alpha)\right)-D_{\varphi}\left(h^{*}(\alpha)\right) .
$$

Further, $D_{F}(\alpha)=0$ for $\alpha<h_{0}$ and $D_{F}(\alpha)=1$ for $\alpha>1$. Thus $F(n) / n$ has a continuous distribution function. 
4. Upper estimates. We shall exploit the observation, based on the graph of $h$, that $F(n) / n$ is near its largest when $\varphi(n) / n$ is near 0 .

LEMmA 1. For all large $x$ there exists an integer $n_{0}=n_{0}(x)$ such that $x-x \log ^{-1} x<n_{0} \leqq x$ and

$$
\varphi\left(n_{0}\right) / n_{0} \sim e^{-r} / \log \log x \sim \min _{1 \leqq m \leq x} \varphi(m) / m .
$$

Proof. Let $p_{r}$ denote the $r$ th prime (in the usual order) and $P(r)$ the product of the first $r$ primes. Choose $r^{\prime}=r^{\prime}(x)$ to be the largest integer for which $P\left(r^{\prime}\right) \leqq x / \log x$. The prime number theorem implies that

$$
\sum_{p \leqq p_{r^{\prime}}} \log p \sim p_{r^{\prime}},
$$

and hence, by an easy calculation, $p_{r^{\prime}} \sim \log x$.

Set $n_{0}=\left[x / P\left(r^{\prime}\right)\right] P\left(r^{\prime}\right)$. Then $x-P\left(r^{\prime}\right)<n_{0} \leqq x$ and

$$
\frac{\varphi\left(n_{0}\right)}{n_{0}} \leqq \prod_{p \leqq p_{r^{\prime}}}\left(1-\frac{1}{p}\right) \sim \frac{e^{-r}}{\log p_{r^{\prime}}} \sim \frac{e^{-r}}{\log \log x} .
$$

It is known (cf. [5, Th. 328]) that

$$
\min _{1 \leqq m \leqq x} \varphi(m) / m \sim e^{-r} / \log \log x .
$$

THEOREM 2. As $x \rightarrow \infty$,

$$
\max _{n \leqq x} F(n)=x-\left(\zeta e^{-r}+o(1)\right) x / \log \log x .
$$

Proof. Let $\alpha_{0}$ (presently to be specified) be a small positive number such that $h(\alpha) \leqq h\left(\alpha_{0}\right)<1$ for $\alpha_{0}<\alpha<1$. Suppose first that $\varphi(n) / n \geqq \alpha_{0}$. Then there exists an $\varepsilon>0$ such that $F(n)<(1-\varepsilon) n$ for all sufficiently large $n$ and if $x$ is large, $F(n)<(1-\varepsilon) x$ for all $n \leqq x$ and satisfying $\varphi(n) / n \geqq \alpha_{0}$.

For small positive values of $\alpha$ we use the approximation

$$
g(\alpha)=\zeta \alpha+O\{\exp (-\exp 1 /(k \alpha))\},
$$

which holds for some absolute constant $k$ [2, Lemma 4]. If we combine this estimate with (1) and (2) we obtain

$$
\frac{F(n)}{n}=1-\zeta \frac{\varphi(n)}{n}+O\left\{\exp \left(-\exp \frac{n}{k \varphi(n)}\right)\right\}+O\left(e^{-\sqrt{\log n}}\right) .
$$

The function $\alpha \mapsto 1-\zeta \alpha+c \exp \{-\exp 1 /(k \alpha)\}$ is decreasing for small positive $\alpha$. Choose $\alpha$, to be positive but so small that the function 
is decreasing for $0<\alpha<\alpha_{0}$ and $h\left(\alpha_{0}\right)>\zeta-1$.

Now for $\varphi(n) / n<\alpha_{0}$ we use the inequality

$$
\varphi(n) / n \geqq\left(e^{-r}+o(1)\right) / \log \log x, \quad 1 \leqq n \leqq x,
$$

to obtain the bound

$$
F(n) \leqq x\left\{1-\left(\zeta e^{-r}+o(1)\right) / \log \log x\right\}, \quad 1 \leqq n \leqq x .
$$

The $o(1)$ term tends to zero as $x \rightarrow \infty$ (independently of $n$ ).

On the other hand, taking $n_{0}$ as in the lemma yields

$$
\begin{aligned}
F\left(n_{0}\right) & =n_{0}\left\{1-\left(\zeta e^{-r}+o(1)\right) / \log \log x\right\} \\
& =x\left\{1-\left(\zeta e^{-r}+o(1)\right) / \log \log x\right\} .
\end{aligned}
$$

Define a sequence $\left\{n_{k}\right\}$ of "new highs" of $F$ by the condition $F(n)<F\left(n_{k}\right)$ for all $n<n_{k}$.

We note for later use that $\varphi\left(n_{k}\right) / n_{k} \sim e^{-r} / \log \log n_{k}$ as $k \rightarrow \infty$. We can see this by noting first that $\varphi\left(n_{k}\right) / n_{k} \rightarrow 0$ by the first paragraph of the proof of Theorem 2. Then we write (4) with $n=n_{k}$ and Theorem 2 with $x=n_{k}$ and equate the expressions to obtain

$$
1-\frac{\zeta \varphi\left(n_{k}\right)}{n_{k}}(1+o(1))+O\left(e^{-\sqrt{\log n}}\right)=1-\frac{\zeta e^{-r}+o(1)}{\log \log n_{k}} .
$$

Theorem 2 has two immediate consequences.

CoROLlaRy 1. $F(n)<n$ for all sufficiently large $n$.

COROLLARY 2.

$$
n_{k+1}-n_{k}=o\left(n_{k} / \log \log n_{k}\right), \quad k \longrightarrow \infty .
$$

Proof. For $n_{k} \leqq x<n_{k+1}$ we have

$$
\max _{n \leqq x} F(n)=F\left(n_{k}\right)
$$

or

$$
x\left\{1-\frac{\zeta e^{-r}+o(1)}{\log \log x}\right\}=n_{k}\left\{1-\frac{\zeta e^{-r}+o(1)}{\log \log n_{k}}\right\} .
$$

Let $x \rightarrow n_{k+1^{-}}$to obtain the corollary.

REMARK. The size of $n$ or $n_{k}$ plays a vital role in the two corollaries. The first corollary is false for small $n$ as the examples $F(13)=13$ and $F(73)=75$ show.

The proof of Theorem 2 implies that $\varphi\left(n_{k}\right) / n_{k} \rightarrow 0$ as $k \rightarrow \infty$. 
Numerical computation shows that the $n_{k}$ 's are primes for all $n_{k} \leqq$ 500 (the limit of the calculation). The explanation of this anomaly (apart from the effect of the error term) is as follows. Let $u_{1}$ be the number in $(0,1)$ for which $h\left(u_{1}\right)=\zeta-1$ (cf. (Fig. 1)). It appears from (4) that $u_{1} \approx .03$. Simple estimates show that $\varphi(n) / n>.03$ for all $n<e^{e^{18}}$. Thus for $n$ of modest size, the largest values of $h(\varphi(n) / n)$ occur for $\varphi(n) / n$ near 1 .

We conclude this section by establishing a lower bound inequality for $n_{k+1}-n_{k}$.

THEOREM 3. For any $\varepsilon>0$

$$
n_{k+1}-n_{k}>n_{k}^{1-\varepsilon}, k \longrightarrow \infty \text {. }
$$

Proof. Given $\varepsilon>0$ and $n_{k}$, let $p^{*}=p^{*}(k)$ denote the largest prime such that $\Pi_{p \leqq p^{*}} p \leqq n_{k}$. The prime number theorem and simple estimates imply that $p^{*} \sim \log n_{k}$. We shall show that at most $\varepsilon p^{*} / \log p^{*}$ primes $p \leqq p^{*}$ fail to divide $n_{k}$. Similar estimates apply for $n_{k+1}$ and thus $n_{k}$ and $n_{k+1}$ have at least $\pi\left(p^{*}\right)-2\left[\varepsilon p^{*} / \log p^{*}\right]$ prime factors in common.

Let $w$ be an integer such that

$$
\pi(w)=\pi\left(p^{*}\right)-2\left[\varepsilon p^{*} / \log p^{*}\right] .
$$

Then we have

$$
n_{k+1}-n_{k} \geqq \prod_{p \leq w} p=\prod_{p \leqq p *} p \prod_{w<p \leqq p^{*}} p^{-1}
$$

Also,

$$
\sum_{w<p \leqq p^{*}} \log p \leqq\left(\log p^{*}\right)\left[\pi\left(p^{*}\right)-\pi(w)\right] \leqq 2 \varepsilon p^{*}
$$

and so

$$
n_{k+1}-n_{k} \geqq \frac{n_{k}}{2 p^{*}} \exp \left[-2 \varepsilon p^{*}\right] \geqq n_{k}^{1-3 \varepsilon} .
$$

We introduce the integer

$$
N=\left[n_{k} \prod_{p<p^{*}} p^{-1}\right] \prod_{p<p^{*}} p
$$

Since $N \leqq n_{k}$ we have $F(N) \leqq F\left(n_{k}\right)$. We can estimate $F(N)$ and $F\left(n_{k}\right)$ because of the special form of $N$ and $n_{k}$. Also, $N$ is not much smaller than $n_{k}$. These facts will enable us to show that

$$
\#\left\{p \leqq p^{*}: p \nmid n_{k}\right\} \leqq \varepsilon p^{*} / \log p^{*} .
$$

Let $\nu$ denote the number of primes $p \leqq p^{*}$ such that $p \nmid n_{k}$. We suppose that $\nu>\varepsilon p^{*} / \log p^{*}$ and shall deduce a contradiction. 
At most $\nu+1$ prime divisors of $n_{k}$ (counting multiplicity) can exceed $p^{*}$, as we now indicate. Suppose that there were at least $\nu+2$ prime divisors of $n_{k}$ exceeding $p^{*}$. For each of the $\nu$ primes $p_{i} \leqq p^{*}$ with $p_{i} \nmid n_{k}$ associate a prime $p_{i}^{\prime}>p^{*}$ with $p_{i}^{\prime} \mid n_{k}$. Each of the $p^{\prime \prime s}$ can be used at most as many times as it occurs in the factorization of $n_{k}$. We have

$$
n_{k}>n^{\prime}=n_{k} \prod_{i=1}^{\nu} p_{i} / p_{i}^{\prime} ;
$$

further $n^{\prime}$ is divisible by each prime not exceeding $p^{*}$ and by at least two primes exceeding $p^{*}$. Thus $n_{k}>n^{\prime}>p^{* 2} \Pi_{p \leq p^{*}} p$. On the other hand the definition of $p^{*}$ implies that $n_{k}<2 p^{*} \Pi_{p \leqq p^{*}} p$, contradicting the last inequality.

Let $y$ and $z$ denote composite numbers such that $\pi\left(p^{*}\right)-\pi(y)=\nu$, $\pi(z)-\pi\left(p^{*}\right)=\nu+1$. Then

$$
\begin{aligned}
\frac{\varphi\left(n_{k}\right)}{n_{k}} & =\prod_{p \leqq p^{*}}\left(1-\frac{1}{p}\right) \prod_{\substack{p \leq p^{*} \\
p \nmid n_{k}}}\left(1-\frac{1}{p}\right)^{-1} \prod_{\substack{p>p^{*} \\
p \eta_{k}}}\left(1-\frac{1}{p}\right) \\
& \geqq \prod_{p \leqq p^{*}}\left(1-\frac{1}{p}\right) \prod_{y<p \leqq p^{*}}\left(1-\frac{1}{p}\right)^{-1} \prod_{p *<p<z}\left(1-\frac{1}{p}\right) .
\end{aligned}
$$

Letting $\nu=\eta p^{*} / \log p^{*}, \varepsilon<\eta \leqq 1$, we have

$$
\pi(y)=\pi\left(p^{*}\right)-\nu=(1-\eta+o(1)) p^{*} / \log p^{*},
$$

and so $y=(1-\eta+o(1)) p^{*}$. Similarly $z=(1+\eta+o(1)) p^{*}$. Thus

$$
\prod_{y<p \leq p^{*}}\left(1-\frac{1}{p}\right)^{-1} \prod_{p^{*}<p<z}\left(1-\frac{1}{p}\right)=\frac{\left(\log p^{*}\right)^{2}}{(\log y)(\log z)}\left(1+O\left(e^{-\sqrt{\log p^{*}}}\right)\right) .
$$

Differentiation shows that, for fixed $q$, the function

$$
\eta \longmapsto \frac{\log ^{2} q}{\log ((1-\eta) q) \log ((1+\eta) q)}
$$

is increasing for $0<\eta<1$. Thus

$$
\begin{aligned}
\frac{\left(\log p^{*}\right)^{2}}{(\log y)(\log z)} & \geqq \frac{\left(\log p^{*}\right)^{2}}{\log \left((1-\varepsilon) p^{*}\right) \log \left((1+\varepsilon) p^{*}\right)} \\
& \geqq\left\{1-\frac{\varepsilon+\varepsilon^{2} / 2+O\left(\varepsilon^{3}\right)}{\log p^{*}}\right\}^{-1}\left\{1+\frac{\varepsilon-\varepsilon^{2} / 2+O\left(\varepsilon^{3}\right)}{\log p^{*}}\right\}^{-1} \\
& \geqq 1+\frac{\varepsilon^{2}}{\log p^{*}}+O\left(\frac{\varepsilon^{3}}{\log p^{*}}+\frac{\varepsilon^{2}}{\log ^{2} p^{*}}\right) .
\end{aligned}
$$

Thus

$$
\prod_{v<p \leqq p^{*}}\left(1-\frac{1}{p}\right)^{-1} \prod_{p^{*}<p<z}\left(1-\frac{1}{p}\right) \geqq 1+\frac{\varepsilon^{2}}{2 \log p^{*}},
$$


provided that $k$ is sufficiently large and $\varepsilon$ sufficiently small. It follows that

$$
\frac{\varphi\left(n_{k}\right)}{n_{k}} \geqq\left(1+\frac{\varepsilon^{2}}{2 \log p^{*}}\right) \prod_{p \leqq p^{*}}\left(1-\frac{1}{p}\right) .
$$

We have $\varphi(N) / N \sim e^{-r} / \log \log N$ because of the form of $N$, and $\varphi\left(n_{k}\right) / n_{k} \sim e^{-r} / \log \log n_{k}$ by the argument following the proof of Theorem 2. It follows from (4), that for some $\alpha>0$,

$$
\frac{F(x)}{x}=1-\zeta \frac{\varphi(x)}{x}+O\left\{\exp \left(-\log ^{\alpha} x\right)\right\}
$$

holds for $x=N$ and $x=n_{k}$.

We combine the formulas for $F\left(n_{k}\right)$ and $F(N)$ with the bound we obtained for $\varphi\left(n_{k}\right) / n_{k}$, the inequalities

$$
n_{k} \geqq N=\left[\frac{n_{k}}{\prod_{p<p^{*}} p}\right] \prod_{p<p^{*}} p>n_{k}-\prod_{p<p^{*}} p \geqq n_{k}\left(1-\frac{1}{p^{*}}\right)
$$

and $\varphi(N) / N \leqq \Pi_{p<p^{*}}\left(1-p^{-1}\right)$ to obtain

$$
\begin{aligned}
F\left(n_{k}\right) & \leqq \frac{N}{1-\frac{1}{p^{*}}}\left\{1-\zeta\left(1+\frac{\varepsilon^{2}}{2 \log p^{*}}\right) \prod_{p \leqq p^{*}}\left(1-\frac{1}{p}\right)+c e^{-\log ^{\alpha} N}\right\} \\
& <N\left\{1-\zeta \prod_{p<p^{*}}\left(1-\frac{1}{p}\right)-c \exp \left(-\log ^{\alpha} N\right)\right\} \leqq F(N),
\end{aligned}
$$

where $c$ is a positive constant. This inequality is impossible, since the $n_{k}$ 's are the new highs of $F$. It follows that at most $\varepsilon p^{*} / \log p^{*}$ primes $p \leqq p^{*}$ fail to divide $n_{k}$ and hence our lower bound for $n_{n+1}-n_{k}$ holds.

5. Small values of $F(n) / n$. We have shown in $\S 2$ that $F(n) / n \sim$ $h(\varphi(n) / n)$. The function $h$ attains a minimal value $h_{0}$ at an interior point $u_{0}$ of $(0,1)$, as we presently shall show. The point $u_{0}$ is unique by the strict convexity of $h$. Thus $F(n) / n$ is, asymptotically, near its minimal value $h_{0}$ when $\varphi(n) / n$ is near $u_{0}$.

Numerical data suggest that $u_{0}$ is near $1 / 2$ and $h_{0}$ is near $1 / 3$. We shall show that $.473<u_{0}<.475$ and $.321<h_{0}<.324$.

Lemma 2. $h^{\prime}(0)=-\zeta, h^{\prime}(1)=\zeta$.

Proof. We have by (1) that $h^{\prime}(u)=\zeta-2 g^{\prime}(u)$. The estimate (cf. [2], Lemma 4)

$$
g(u)=\zeta u+O\{\exp (-\exp 1 /(k u))\}
$$


implies that $g^{\prime}(0)=\zeta$, and hence $h^{\prime}(0)=-\zeta$. Equation $(0)$ implies that $g^{\prime}(1)=0$, and hence $h^{\prime}(1)=\zeta$.

Thus the minimum of $h$ is achieved in the open interval $(0,1)$.

We shall now establish a formula which will lead to estimates for $g(1 / 2)$. This will be useful because of the close connection between $g$ and $h$ and the proximity of $u_{0}$ to $1 / 2$.

LEMMA 3.

$$
\begin{aligned}
g(1 / 2)=\frac{1}{2}+\frac{\zeta}{6} & -\left\{\left(\frac{\zeta}{4}-g\left(\frac{1}{4}\right)\right)-\left(\frac{\zeta}{8}-g\left(\frac{1}{8}\right)\right)\right. \\
+ & \left.\left(\frac{\zeta}{16}-g\left(\frac{1}{16}\right)\right)-\cdots\right\} .
\end{aligned}
$$

Proof. We estimate

$$
\#\{n \leqq x: n \text { odd, } \varphi(n) \leqq y\},
$$

a problem closely related to the main theorem of [2]. The generating function

$$
\begin{aligned}
F(s, z) & \stackrel{\text { def }}{=} \sum_{n=1}^{\infty} n^{-s} \varphi(n)^{-z} \\
& =\prod_{p}\left\{1+p^{-s}(p-1)^{-s}\left(1+p^{-s-z}+p^{-2 s-2 z}+\cdots\right)\right\} \\
& =\prod_{p}\left\{1-p^{-s-z}+p^{-s}(p-1)^{-z}\right\} \zeta(s+z) \\
& \stackrel{\text { def }}{=} \Pi(s, z) \zeta(s+z)
\end{aligned}
$$

was used in [2], and the function $g$ was represented by

$$
g(\alpha)=\frac{1}{2 \pi i} \int_{1 / 2-i \infty}^{1 / 2+i \infty} \frac{\Pi(1-z, z)}{z(1-z)} \alpha^{z} d z, \quad 0 \leqq \alpha \leqq 1
$$

The formula is valid at the end points by uniform convergence of the integral.

We delete the even integers and write

$$
\begin{aligned}
F_{0}(s, z) & =\sum_{\substack{n=1 \\
n \text { odd }}}^{\infty} n^{-s} \varphi(n)^{-z} \\
& =\Pi(s, z) \zeta(s+z)\left\{\frac{1-2^{-s-z}}{1-2^{-s-z}+2^{-s}}\right\} .
\end{aligned}
$$

The functions $F(s, z)$ and $F_{0}(s, z)$ have the same singularities in the region

$$
\{(s, z) \in C \times C: \operatorname{Re} s+z>0\}
$$


because any singularity of the new factor $\left(1-2^{-s-z}\right) /\left(1-2^{-s-z}+2^{-s}\right)$ is cancelled by a zero of $\Pi(s, z)$, and the new factor has no zeros in this region.

It now follows, mutatis mutandis, that

$$
\begin{aligned}
g_{0}(\alpha) & \stackrel{\text { def }}{=} \lim _{x \rightarrow \infty} \frac{1}{x} \sharp\{n \leqq x: n \text { odd, } \varphi(n) \leqq \alpha x\} \\
& =\frac{1}{2 \pi i} \int_{1 / 2-i \infty}^{1 / 2+i \infty} \frac{\Pi(1-z, z)}{z(1-z)} \alpha^{z}\left(1+2^{z}\right)^{-1} d z \\
& =\frac{1}{2 \pi i} \int_{1 / 2-i \infty}^{1 / 2+i \infty} \frac{\Pi(1-z, z)}{z(1-z)}\left\{\left(\frac{\alpha}{2}\right)^{z}-\left(\frac{\alpha}{4}\right)^{z}+\left(\frac{\alpha}{8}\right)^{z}-\cdots\right\} d z \\
& =g(\alpha / 2)-g(\alpha / 4)+g(\alpha / 8)-\cdots .
\end{aligned}
$$

If we note that $g_{0}(1)=1 / 2$ and sum the series $\zeta / 4-\zeta / 8+\zeta / 16-\cdots$ we obtain the lemma.

Now $g$ is concave and $g(\varepsilon) \sim \zeta \varepsilon$ as $\varepsilon \rightarrow 0$. Thus the series in the formula for $g(1 / 2)$ is alternating with terms decreasing to zero, indeed at a geometric rate. To further exploit our formula we must first estimate $D_{\varphi}(t)$ for $t$ near 0 .

Lemma $4 . \quad D_{\varphi}(t)<12 t^{3}, 0<t<1$.

Proof. By Chebychev's inequality

$$
t^{-3} \sharp\left\{n \leqq x: \frac{\varphi(n)}{n} \leqq t\right\}=t^{-3} \sum_{\substack{n \leq x \\ n \mid \varphi(n) \geq 1 / t}} 1 \leqq \sum_{n \leqq x}\left(\frac{n}{\varphi(n)}\right)^{3},
$$

and we estimate the last sum by writing

$$
(n / \varphi(n))^{3}=(1 * \beta)(n),
$$

where $*$ denotes multiplicative convolution and $\beta$ is a nonnegative multiplicative function satisfying $\beta(p)=\left(p^{3}-(p-1)^{3}\right) /(p-1)^{3}, \beta\left(p^{\alpha}\right)=0$ for all primes $p$ and all exponents $\alpha \geqq 2$.

Thus

$$
\begin{aligned}
& \sum_{n \leqq x}\left(\frac{n}{\varphi(n)}\right)^{3}=\sum_{n \leqq x}\left[\frac{x}{n}\right] \beta(n) \\
& \leqq x \sum_{n=1}^{\infty} \frac{\beta(n)}{n}=x \prod_{p}\left(1+\frac{\beta(p)}{p}\right) \\
& \quad x \prod_{p}\left\{1+\frac{1}{p} \frac{p^{3}-(p-1)^{3}}{(p-1)^{3}}\right\} \stackrel{\text { def }}{=} \gamma x .
\end{aligned}
$$

Now 


$$
\begin{aligned}
\gamma & =\zeta(2)^{3} \prod_{p}\left\{1+\frac{3 p^{2}-3 p+1}{p(p-1)^{3}}\right\}\left\{1-\frac{1}{p^{2}}\right\}^{3} \\
& =\zeta(2)^{3} \prod_{p}\left\{1+\frac{6 p^{4}+4 p^{3}-3 p^{2}-p+1}{p^{7}}\right\} .
\end{aligned}
$$

It is easy to check that for all $p \geqq 3$

$$
6 p^{4}+4 p^{3}-3 p^{2}-p+1<7 p^{4} \text {. }
$$

We have

$$
\gamma \leqq \zeta(2)^{3}\left(1+\frac{115}{128}\right)\left\{\left(1+\frac{7}{3^{3}}\right)\left(1+\frac{7}{5^{3}}\right)\left(1+\frac{7}{7^{3}}\right)\right\} \exp \left\{\sum_{p \geqq 11} 7 p^{-3}\right\},
$$

and

$$
7 \sum_{p \geqq 11} p^{-3}<7 \int_{10}^{\infty} t^{-3} d t=.035
$$

Thus $\gamma \leqq 12$, and $D_{\varphi}(t)$ satisfies the claimed bound.

We combine the last two lemmas with numerical data of Charles R. Wall [10] on the density function $D_{\varphi}$ to obtain upper and lower estimates for $g(1 / 2)$.

LEMMA 5.

$$
\frac{1}{2}+\frac{\zeta}{6}-.00154<g(1 / 2)<\frac{1}{2}+\frac{\zeta}{6}-.00075 .
$$

Proof. The alternating series representation of $g(1 / 2)$ leads to the inequalities

$$
\begin{aligned}
\frac{1}{2}+ & \frac{\zeta}{6}-\left\{\left(\frac{\zeta}{4}-g\left(\frac{1}{4}\right)\right)-\left(\frac{\zeta}{8}-g\left(\frac{1}{8}\right)\right)+\left(\frac{\zeta}{16}-g\left(\frac{1}{16}\right)\right)\right\} \\
& \leqq g(1 / 2) \leqq \frac{1}{2}+\frac{\zeta}{6}-\left\{\left(\frac{\zeta}{4}-g\left(\frac{1}{4}\right)\right)-\left(\frac{\zeta}{8}-g\left(\frac{1}{8}\right)\right)\right\}
\end{aligned}
$$

The differential equation (0) has the solution

$$
u^{-1} g(u)=\zeta-\int_{0}^{u} D_{\varphi}(t) t^{-2} d t
$$

The constant is evaluated here by noting that $g^{\prime}(0)=\zeta$. The integral converges at zero by the preceding lemma. Thus we have

$$
2^{-k \zeta}-g\left(2^{-k}\right)=2^{-k} \int_{0}^{2^{-k}} D_{\varphi}(t) t^{-2} d t
$$

It follows that 


$$
\begin{aligned}
\left(\frac{\zeta}{4}-\right. & \left.g\left(\frac{1}{4}\right)\right)-\left(\frac{\zeta}{8}-g\left(\frac{1}{8}\right)\right)+\left(\frac{\zeta}{16}-g\left(\frac{1}{16}\right)\right) \\
& =\frac{1}{4} \int_{1 / 8}^{1 / 4} D_{\varphi}(t) \frac{d t}{t^{2}}+\frac{1}{8} \int_{1 / 16}^{1 / 8} D_{\varphi}(t) \frac{d t}{t^{2}}+\frac{3}{16} \int_{0}^{1 / 16} D_{\varphi}(t) \frac{d t}{t^{2}} .
\end{aligned}
$$

We estimate the three integrals from above, using the bound of the preceding lemma for $0 \leqq t \leqq .007$ and the upper bounds of Wall for $.007<t \leqq .25$. We obtain the upper bound .00154 .

Similar treatment of

$$
\left(\frac{\zeta}{4}-g\left(\frac{1}{4}\right)\right)-\left(\frac{\zeta}{8}-g\left(\frac{1}{8}\right)\right)
$$

leads to the lower bound .00075 .

LEMMA 6. (Main formula.)

$$
2 D_{\varphi}(1 / 2)-1+\zeta / 6+2 R=\int_{u_{0}}^{1 / 2} t^{-1} d D_{\varphi}(t),
$$

where $.00075<R<.00154$.

Proof. We have by (5)

$$
\frac{g\left(u_{0}\right)}{u_{0}}-\frac{g(1 / 2)}{1 / 2}=\int_{u_{0}}^{1 / 2} D_{\varphi}(t) t^{-2} d t
$$

From (1) and the fact that $h^{\prime}\left(u_{0}\right)=0$ we get $g^{\prime}\left(u_{0}\right)=\zeta / 2$. Combining this with $(0)$ we obtain

$$
g\left(u_{n}\right)=u_{0} \zeta / 2+D_{\varphi}\left(u_{0}\right)
$$

This expression, Lemma 5, and the preceding integral yield

$$
\frac{D_{\varphi}\left(u_{0}\right)}{u_{0}}-1+\frac{\zeta}{6}+2 R=\int_{u_{0}}^{1 / 2} D_{\varphi}(t) t^{-2} d t .
$$

Integrating by parts we get the desired expression.

THEOREM 4. $u_{0}>.473$ and $h_{0}<.324$.

Proof. Starting from Lemma 6, we write

$$
\begin{aligned}
& 2 D_{\varphi}\left(\frac{1}{2}\right)-1+\frac{\zeta}{6}+2 R=\left\{\int_{.475}^{.5}+\int_{u_{0}}^{.475}\right\} t^{-1} d D_{\varphi}(t) \\
& \geqq \frac{1}{.5}\left\{D_{\varphi}(.5)-D_{\varphi}(.499)\right\}+\frac{1}{.499}\left\{D_{\varphi}(.499)-D_{\varphi}(.498)\right\} \\
&+\cdots+\frac{1}{.476}\left\{D_{\varphi}(.476)-D_{\varphi}(.475)\right\}+\frac{1}{.475}\left\{D_{\varphi}(.475)-D_{\varphi}\left(u_{0}\right)\right\},
\end{aligned}
$$


A MEASURE OF THE NONMONOTONICITY OF THE EULER PHI FUNCTION 95

Note that this inequality is valid regardless of whether $u_{0} \leqq .475$ or not.

We rearrange terms, isolating $D_{\varphi}\left(u_{0}\right)$ :

$$
\begin{gathered}
\frac{D_{\varphi}\left(u_{0}\right)}{.475} \geqq 1-\frac{\zeta}{6}-2 R+\left(\frac{1}{.499}-\frac{1}{.5}\right) D_{\varphi}(.499) \\
+\cdots+\left(\frac{1}{.475}-\frac{1}{.476}\right) D_{\varphi}(.475)
\end{gathered}
$$

If we use the upper estimate for $R$ and the lower estimates of [10] for $D_{\varphi}(.475), \cdots, D_{\varphi}(.499)$, we find that $D_{\varphi}\left(u_{0}\right)>.3380$.

The stated inequalities follow at once from this bound. First, we have from [10] that $D_{\varphi}(.473)<.3362$, and thus $u_{0}>.473$. Next, it follows from Equations (0) and (1) that $h_{0}=1-2 D_{\varphi}\left(u_{0}\right)$. Thus, $h_{0}<.324$.

We also have bounds for $u_{0}$ and $h_{0}$ in the opposite directions.

THEOREM 5. $u_{0}<.475$ and $h_{0}>.321$.

Proof. Using Lemma 6 again, we write

$$
2 D_{\varphi}\left(\frac{1}{2}\right)-1+\frac{\zeta}{6}+2 R=\left\{\int_{.475}^{.5}+\int_{u_{0}}^{.475}\right\} t^{-1} d D_{\varphi}(t)
$$

This time we express the first integral as an upper Riemann-Stieltjes sum and sum by parts to obtain

$$
\begin{aligned}
\int_{.475}^{.5} t^{-1} d D_{\varphi}(t) \leqq & \frac{D_{\varphi}(.5)}{.499}+\left(\frac{1}{.498}-\frac{1}{.499}\right) D_{\varphi}(.499) \\
& +\cdots+\left(\frac{1}{.475}-\frac{1}{.476}\right) D_{\varphi}(.476)-\frac{D_{\varphi}(.475)}{.475} .
\end{aligned}
$$

Thus

$$
\int_{u_{0}}^{.475} t^{-1} d D_{\varphi}(t) \geqq \frac{D_{\varphi}(.475)}{.475}-I
$$

where

$I=1-\frac{\zeta}{6}-2 R+\left(\frac{1}{.499}-\frac{1}{.5}\right) D_{\varphi}(.5)+\cdots+\left(\frac{1}{.475}-\frac{1}{.476}\right) D_{\varphi}(.476)$.

We estimate $I$ from above by using the upper bounds for $D_{\varphi}(.476), \cdots, D_{\varphi}(.500)$ from [10] and the lower bound for $R$ from Lemma 6 . We obtain the inequality

$$
\int_{u_{0}}^{.475} t^{-1} d D_{\varphi}(t) \geqq \frac{D_{\varphi}(.475)}{.475}-.7145,
$$


from which both assertions of the theorem will follow.

The bound $D_{\varphi}(.475) \geqq .33969$ from [10] implies that

$$
\int_{u_{0}}^{.475} t^{-1} d D_{\varphi}(t)>.0006>0
$$

and hence $u_{0}<.475$.

Next, since $u_{0}>.473$, we obtain from (6)

$$
\frac{1}{.473}\left\{D_{\varphi}(.475)-D_{\varphi}\left(u_{0}\right)\right\} \geqq \frac{D_{\varphi}(.475)}{.475}-.7145 \text {. }
$$

This inequality and the bound $D_{\varphi}(.475)<.34166$ from [10] yield $D_{\varphi}\left(u_{0}\right)<.3394$. Thus, we finally obtain $h_{0}=1-2 D_{\varphi}\left(u_{0}\right)>.321$.

6. Lower estimates for $F$. The sequence $F(n)$ tends to infinity with $n$, since

$$
F(n) / n \sim h(\varphi(n) / n) \geqq h_{0}>0 .
$$

In this section we are going to establish

TheOREM 6. As $x \rightarrow \infty$,

$$
\min _{n>x} F(n) \sim h_{0} x
$$

This estimate follows easily from the following

Lemma 7. Let $\alpha \in(0,1)$ and let $\varepsilon>0$ be given. Then there exists an $X$ (depending on $\varepsilon$ and $\alpha$ ) such that for each $x \geqq X$, the interval $(x, x+\varepsilon x]$ contains an integer $j$ with $|\varphi(j) / j-\alpha|<\varepsilon$.

Proof. The argument proceeds in two steps. First we obtain some integer $j_{0}$ (not necessarily in $(x, x+\varepsilon x]$ ) composed of at least two distinct prime factors, for which $\left|\varphi\left(j_{0}\right) / j_{0}-\alpha\right|<\varepsilon$. Then we show that a suitable multiple of $j_{0}$ lies in $(x, x+\varepsilon x]$ and satisfies the same $\varphi$ estimate.

Let $\alpha=\alpha_{0}$. Let $q_{1}$ be the smallest prime $p_{\nu}$ for which $1-$ $p_{\nu}^{-1}>\alpha_{0}$. Set $\alpha_{1}=\alpha_{0}\left(1-q_{1}^{-1}\right)^{-1}$ and $j_{1}=q_{1}$. Repeat the foregoing, choosing $q_{2}$ to be the smallest prime $p_{\nu}$ exceeding $q_{1}$ for which $1-p_{\nu}^{-1}>\alpha_{1}$. Let $j_{2}=q_{1} q_{2}$ and $\alpha_{2}=\alpha_{1}\left(1-q_{2}^{-1}\right)^{-1}$. If $1>\alpha_{2}>1-$ $\varepsilon /(\alpha+\varepsilon)$, we can stop here. Otherwise we continue until we obtain an integer $j_{r}=q_{1} q_{2} \cdots q_{r}, r=r(\alpha, \varepsilon)$, such that

$$
\alpha \leqq \varphi\left(j_{r}\right) / j_{r}<\alpha+\varepsilon \text {. }
$$

This is possible to achieve since $1-p_{\nu}^{-1} \rightarrow 1$ as $\nu \rightarrow \infty$ and $\Pi_{\nu=1}^{\infty}\left(1-p_{\nu}^{-1}\right)=0$. 
Set $j_{\nu}=j^{*}$ and consider the sequence $\left\{j^{*} q_{1}^{a} q_{2}^{b}: a, b=0,1,2,3, \cdots\right\}$. Clearly

$$
\varphi\left(j^{*}\right) / j^{*}=\varphi\left(j^{*} q_{1}^{a} \alpha_{2}^{b}\right) /\left(j^{*} q_{1}^{a} q_{2}^{b}\right) .
$$

It suffices to show that for each large $x$ the interval $(x, x+\varepsilon x]$ contains some $q_{1}^{a} q_{2}^{b}, a, b \geqq 0$.

It is well known that the sequence $\left\{q_{1}^{a} q_{2}^{b}: a, b \in \boldsymbol{Z}\right\}$ is dense in the positive reals for $q_{1}, q_{2}$ distinct primes. Choose $a>0$ and $-b<0$ such that $1<q_{1}^{a} q_{2}^{-b}<1+\varepsilon$. Given $x$, set

$$
\begin{aligned}
& s=\left[(\log x) /\left(\log q_{1} q_{2}\right)\right], \\
& t=\left[\left(\log q_{1} q_{2}\right) /\left(\log q_{1}^{a} q_{2}^{-b}\right)\right]+1,
\end{aligned}
$$

and $a_{k}=q_{1}^{s+k a} q_{2}^{s-k b},(0 \leqq k \leqq t)$.

We have

and

$$
a_{0}=\left(q_{1} q_{2}\right)^{s} \leqq x<\left(q_{1} q_{2}\right)^{s+1}<a_{t}
$$

$$
1<a_{k+1} / a_{k}=q_{1}^{a} q_{2}^{-b}<1+\varepsilon .
$$

Thus there exists some $k \in[1, t]$ such that $x<q_{1}^{s+k a} q_{2}^{s-k b}<x+\varepsilon x$.

Finally, we must insure that the exponent $s-k b \geqq 0$. This we do by noting that $a, b$, and $t$ depend only on $\varepsilon$ and are fixed, while $s \rightarrow \infty$ with $x$.

LEMma 8. Given $\varepsilon>0$ there exists an $X=X(\varepsilon)$ such that for each $x \geqq X$ the interval $(x, x+\varepsilon x]$ contains an integer $j$ with $h(\varphi(j) / j)<h_{0}+2 \varepsilon$.

Proof. Since $h$ is convex and differentiable we have

$$
\left|h^{\prime}(x)\right| \leqq \max \left\{\left|h^{\prime}(0)\right|,\left|h^{\prime}(1)\right|\right\}=\zeta, \quad 0 \leqq x \leqq 1 .
$$

The mean value theorem and Lemma 7 imply that there exists an integer $j$ in each far out interval $(x, x+\varepsilon x]$ such that

$$
\left|h(\varphi(j) / j)-h_{0}\right| \leqq \zeta\left|\frac{\varphi(j)}{j}-u_{0}\right|<\zeta \varepsilon<2 \varepsilon
$$

Proof of Theorem 6. On the one hand,

$$
\begin{aligned}
\min _{n>x} F(n) & =\min _{n>x}\left\{n h(\varphi(n) / n)+O\left(n e^{-\sqrt{\log n}}\right)\right\} \\
& \geqq x h_{0}-c x e^{-\sqrt{\log x}}=h_{0} x+o(x) .
\end{aligned}
$$

On the other hand, for given $\varepsilon>0$ and all sufficiently large $x$ there exists an integer $m$ such that 


$$
x<m \leqq x+\varepsilon x, \quad h(\varphi(m) / m)<h_{0}+2 \varepsilon .
$$

For this integer $m$ we have

$$
F(m)<\left(h_{0}+2 \varepsilon\right) m+c m e^{-\sqrt{\log m}},
$$

and hence

$$
\begin{aligned}
\min _{n>x} F(n) & \leqq F(m) \leqq\left(h_{0}+2 \varepsilon\right)(x+\varepsilon x)+2 c x e^{-\sqrt{\log x}} \\
& \leqq h_{0} x+o(x) .
\end{aligned}
$$

Let $\left\{m_{k}\right\}_{k=1}^{\infty}$ be the sequence of discontinuities of $x \mapsto \min _{n>x} F(n)$. (Set $m_{1}=2$.) We can deduce from Theorem 6 the following

COROLLARY 3. $m_{k+1} / m_{k} \rightarrow 1$ as $k \rightarrow \infty$.

Proof. For $m_{k} \leqq x<m_{k+1}$ we have

$$
\min _{n>m_{k}} F(n)=\min _{n>x} F(n) \text {. }
$$

Thus $h_{0} m_{k} \sim h_{0} x$. Let $x \rightarrow m_{k+1}-$.

7. General arithmetic functions. We conclude by showing that rather general arithmetic functions $\psi$ possess an associated monotonicity measuring function $F=F_{\psi}$. Our argument is related to one occurring in [4]. It appears unlikely that there are general analogues of our numbered theorems in $\S \S 3-6$ which are valid without more specific arithmetic information.

It is convenient to estimate the two components of $F$ separately. Let

$$
\begin{aligned}
& F_{1}(n)=\sharp\{m<n: \psi(m) \geqq \psi(n)\}, \\
& F_{2}(n)=\sharp\{m>n: \psi(m) \leqq \psi(n)\} .
\end{aligned}
$$

In both cases we assume that $\psi$ is positive valued and that $\psi(n) / n$ has a distribution function $D_{\psi}$.

TheOREM 7. Let $\psi$ be as above. Then, as $n \rightarrow \infty$,

$$
F_{1}(n)=\psi(n) \int_{t=\psi(n) / n}^{\infty}\left\{1-D_{\psi}(t)\right\} t^{-2} d t+o(n) .
$$

Further, assume that there exist positive numbers $c$ and $\delta$ such that

$$
\#\{m \in(x, 2 x]: \psi(m) / m<y\} \leqq c x y^{1+\delta}
$$

holds for all $y \in(0,1)$ and all $x \geqq 1$. Then 


$$
F_{2}(n)=\psi(n) \int_{t=0}^{\psi(n) / n} D_{\psi}(t) t^{-2} d t+o(n+\psi(n))
$$

REMARKs. A. It is a simple consequence of hypothesis (8) that there exist at most a finite number of integers $n$ for which $\psi(n)$ assumes any one value. Also, (8) implies that the integral in (9) converges at the origin.

B. For application to the Euler $\phi$ function, the estimate

$$
\sum_{m=1}^{n}(m / \psi(m))^{2} \ll n
$$

(cf. [4]) guarantees that (8) holds with $\delta=1$. Condition (8) is vacuous for the sum of divisors function $\sigma$, since $\sigma(n) \geqq n$ for all $n \geqq 1$.

C. Can we replace the equal sign in (7) or in (9) by " " and drop the 0 -term? This is not generally permissible for (7) as one can see by the case in which $D_{\psi}(\alpha)=1$ for some finite $\alpha, \psi(n) / n \geqq \alpha$, and there exists at least one integer $m<n$ such that $\psi(m) \geqq \psi(n)$. The conjecture is also generally false for (9) as well, as we can see in the case where $D_{\varphi}(t)>0$ for all $t>0$. By Remark A there exists an infinite number of integers $n$ for which $F_{2}(n)=0$, and for these $n$ the asymptotic relation would fail.

Proof. We shall show that (9) holds. The proof of (7) is similar but simpler, and is omitted.

Proof. We introduce a partition of $(n, \infty)$. Let $\varepsilon>0, K \in Z^{+}$ with $\varepsilon K>1$ and let $n^{\prime}=n+\psi(n)$. Write

$$
(n, \infty)=\bigcup_{i=1}^{K}\left(n+(i-1) \varepsilon n^{\prime}, n+i \varepsilon n^{\prime}\right] \cup\left(n+K \varepsilon n^{\prime}, \infty\right) .
$$

For the finite intervals we use the following estimates, which are valid for $1 \leqq x<y<\infty$ :

$$
\begin{aligned}
\sharp\{m & \in(x, y]: \psi(m) \leqq m \psi(n) / y\} \\
& \leqq \# \stackrel{\text { def }}{=} \sharp\{m \in(x, y]: \psi(m) \leqq \psi(n)\} \\
& \leqq \#\{m \in(x, y]: \psi(m) \leqq m \psi(n) / x\},
\end{aligned}
$$

and hence

$$
(y-x) D_{\psi}(\psi(n) / y)+o(y) \leqq \# \leqq(y-x) D_{\psi}(\psi(n) / x)+o(y) .
$$

If we set

$$
\Sigma=\varepsilon n^{\prime} \sum_{i=1}^{K} D_{\psi}\left(\psi(n) /\left(n+i \varepsilon n^{\prime}\right)\right)
$$


and

$$
F_{2}(a, b)=\#\{m \in(a, a+b]: \psi(m) \leqq \psi(n)\},
$$

then we obtain

$$
\begin{aligned}
\Sigma+ & K o\left(K \varepsilon n^{\prime}\right) \leqq F_{2}\left(n, K \varepsilon n^{\prime}\right) \\
& \leqq \\
& \quad+K n^{\prime} D_{\psi}(\psi(n) / n)-\varepsilon n^{\prime} D_{\psi}\left(\psi(n) /\left(n+K \varepsilon n^{\prime}\right)\right)
\end{aligned}
$$

Now $\Sigma$ is an approximating sum for the Riemann integral

$$
\begin{aligned}
I & =\varepsilon n^{\prime} \int_{t=0}^{K} D_{\psi}\left(\psi(n) /\left(n+t \varepsilon n^{\prime}\right)\right) d t \\
& =\psi(n) \int_{s=\psi(n) /\left(n+K \varepsilon n^{\prime}\right)}^{\psi(n) / n} D_{\psi}(s) s^{-2} d s,
\end{aligned}
$$

and since the integrand in the first expression is monotone, we get $\left|I-\sum\right|<\varepsilon n^{\prime}$. The hypotheses on $\psi(n) / n$ imply that

$$
D_{\psi}(y) \leqq C y^{1+\delta}, \quad 0<y<1 .
$$

Thus

$$
\int_{0}^{\psi(n) /\left(n+K \varepsilon n^{\prime}\right)} D_{\psi}(t) t^{-2} d t \leqq \frac{C}{\delta}\left(\frac{\psi(n)}{n+K \varepsilon n^{\prime}}\right)^{\delta} \leqq \frac{C}{\delta}(K \varepsilon)^{-\delta} .
$$

Combining these estimates we find that

$$
\begin{aligned}
F_{2}\left(n, K \varepsilon n^{\prime}\right)= & \psi(n) \int_{0}^{\psi(n) / n} D_{\psi}(t) t^{-2} d t \\
& +O\left(\varepsilon n^{\prime}\right)+K o\left(K \varepsilon n^{\prime}\right)+O\left((K \varepsilon)^{-\delta} n^{\prime}\right) .
\end{aligned}
$$

Now we treat the unbounded interval. For each $x \geqq 1$ we have

$$
\begin{aligned}
F_{2}(x, x) & \leqq \#\{m \in(x, 2 x]: \psi(m) / m \leqq \psi(n) / x\} \\
& \leqq C x(\psi(n) / x)^{1+\delta} .
\end{aligned}
$$

Thus

$$
\begin{aligned}
F_{2}\left(n+K \varepsilon n^{\prime}, \infty\right) & \leqq C \psi(n)^{1+\delta}\left(n+K \varepsilon n^{\prime}\right)^{-\delta}\left(1+2^{-\delta}+4^{-\delta}+\cdots\right) \\
& \ll \psi(n)(K \varepsilon)^{-\delta} .
\end{aligned}
$$

It follows that

$$
\begin{aligned}
F_{2}(n)= & \psi(n) \int_{0}^{\psi(n) / n} D_{\psi}(t) t^{-2} d t \\
& +O\left(\varepsilon n^{\prime}\right)+K^{2} \varepsilon o\left(n^{\prime}\right)+O\left((K \varepsilon)^{-\delta} n^{\prime}\right) .
\end{aligned}
$$

If we first choose $\varepsilon$ small and then $K$ so large that $(K \varepsilon)^{-\delta}$ is small, we obtain the desired asymptotic. 


\section{REFERENCES}

1. Paul T. Bateman, The distribution of values of the Euler function, Acta Arith., 21 (1972), 329-345.

2. Harold G. Diamond, The distribution of values of Euler's phi function, Analytic Number Theory P.S.P.M., 24, Amer. Math. Soc., Providence, R.I., (1973), 63-75.

3. Robert E. Dressler, A density which counts multiplicity, Pacific J. Math., 34 (1970), 371-378.

4. Paul Erdös, Some remarks on Euler's $\phi$ function and some related problems, Bull. Amer. Math. Soc., 51 (1945), 540-544.

5. G. H. Hardy and E. M. Wright, An introduction to the theory of numbers, 4th Ed., Oxford Univ. Press, London, 1960.

6. Mark Kac, Statistical independence in probability, analysis, and number theory, Carus Math. Monographs 12, Math. Assn. of America, J. Wiley, New York, 1959.

7. I. J. Schoenberg, Uber die asymptotische Verteilung reeler Zahlen mod 1, Math. Zeit., 28 (1928), 171-199.

8. On asymptotic distributions of arithmetical functions, Trans. Amer. Math. Soc., 39 (1936), 315-330.

9. Charles R. Wall, Density bounds for Euler's function, Math. Comp., 26 (1972), 779-783.

10. $\longrightarrow$ Private communication.

Received July 27, 1977. Research by the first author was supported in part by a grant from the National Science Foundation.

UNIVERSITY OF ILLINOIS

URBANA, IL 61801

AND

Hungarian Academy of Sciences

Budapest, Hungary 



\section{PACIFIC JOURNAL OF MATHEMATICS}

\section{EDITORS}

RICHARD ARENS (Managing Editor)

University of California

Los Angeles, California 90024

C. W. Curtis

University of Oregon

Eugene, OR 97403

C. C. MOORE

University of California

Berkeley, CA 94720

\section{J. DUGUNDJI}

Department of Mathematics University of Southern California Los Angeles, California 90007

R. Finn AND J. Milgram Stanford University Stanford, California 94305

\section{ASSOCIATE EDITORS}

E. F. BeCK ENBACH

B. H. NeUMaNN

F. WOLF

K. Yoshida

\section{SUPPORTING INSTITUTIONS}

UNIVERSITY OF BRITISH COLUMBIA CALIFORNIA INSTITUTE OF TECHNOLOGY UNIVERSITY OF CALIFORNIA MONTANA STATE UNIVERSITY UNIVERSITY OF NEVADA, RENO NEW MEXICO STATE UNIVERSITY OREGON STATE UNIVERSITY UNIVERSITY OF OREGON
UNIVERSITY OF SOUTHERN CALIFORNIA STANFORD UNIVERSITY UNIVERSITY OF HAWAII UNIVERSITY OF TOKYO UNIVERSITY OF UTAH WASHINGTON STATE UNIVERSITY UNIVERSITY OF WASHINGTON 


\section{Pacific Journal of Mathematics \\ Vol. 77, No. $1 \quad$ January, 1978}

Dan Amir, Chebyshev centers and uniform convexity ............... 1

Lawrence Wasson Baggett, Representations of the Mautner group. I ..... 7

George Benke, Trigonometric approximation theory in compact totally

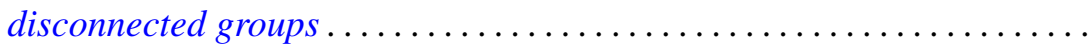

M. Bianchini, O. W. Paques and M. C. Zaine, On the strong compact-ported topology for spaces of holomorphic mappings ..................

Marilyn Breen, Sets with $(d-2)$-dimensional kernels

J. L. Brenner and Allen Kenneth Charnow, Free semigroups of $2 \times 2$

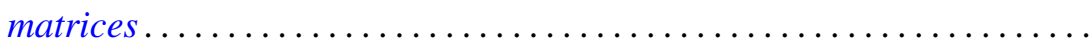

David Bressoud, A new family of partition identities .................

David Fleming Dawson, Summability of matrix transforms of stretchings

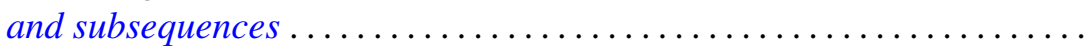

Harold George Diamond and Paul Erdôs, A measure of the nonmonotonicity

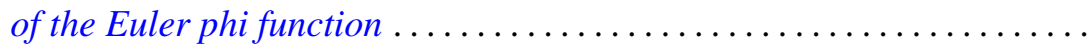

Gary Doyle Faulkner and Ronald Wesley Shonkwiler, Kernel dilation in reproducing kernel Hilbert space and its application to moment

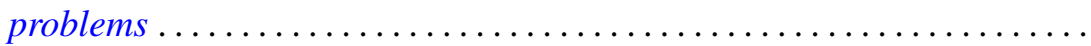

Jan Maksymilian Gronski, Classification of closed sets of attainability in the

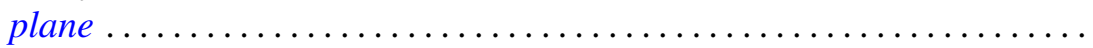

H. B. Hamilton and T. E. Nordahl, Semigroups whose lattice of congruences

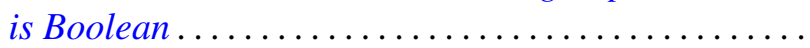

Harvey Bayard Keynes and D. Newton, Minimal $(G, \tau)$-extensions ...

Anthony To-Ming Lau, The Fourier-Stieltjes algebra of a topological

semigroup with involution.

B. C. Oltikar and Luis Ribes, On prosupersolvable groups ...

Brian Lee Peterson, Extensions of pro-affine algebraic groups ...

Thomas M. Phillips, Primitive extensions of Aronszajn spaces ...

Mehdi Radjabalipour, Equivalence of decomposable and 2-decomposable operators. .

M. Satyanarayana, Naturally totally ordered semigroups .

Fred Rex Sinal, A homeomorphism classification of wildly imbedded two-spheres in $S^{3}$

Hugh C. Williams, Some properties of a special set of recurring

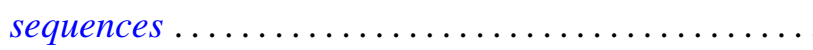

